\title{
ENDOMETRIOSIS : ENIGMATICAL DIAGNOSIS
}

\section{Dr Sandeep Pophale* \\ Dr Shreya Chinchoriya,}

\author{
M.S. Obstetrics and Gynaecology, Asssociate Professor ,Minimal Access \\ Surgery , I.V.F. *Corresponding Author
}

M.S. Obstetrics and Gynaecology, Senior Resident. Dept of Obstetrics and Gynaecology , Tertiary care centre, Mumbai.

ABSTRACT

Background: The prevalence of endometriosis is seen to be from $10 \%$ to $20 \%$ in the reproductive age group whereas that of endometriosis is $30 \%$ to $70 \%$ among women presenting with infertility. In our study the incidence of endometriosis appears to be $6.31 \%$. The objective is study the common presentation in cases of endometriosis and their diagnostic accuracy with the help of radiological modalities . laparoscopy and histopathology remains the gold standard modality to diagnose endometriosis.

Methods: A total of 200 patients with adnexal masses in the women of reproductive age group were studied. Endometriosis was identified by laparoscopy in 12 cases and confirmed by histopathological examination.

Results: Out of the above 200 patients, $6.31 \%$ ( 12 cases) were diagnosed as cases of endometriosis. Out of which, $25 \%$ patients had moderate to severe endometriosis.

The ultrasound turned out to be an important method in diagnosing the cases of endometriosis

Conclusions: The symptoms and clinical signs should raise the suspicion of pelvic endometriosis.ultrasound plays an important role in diagnosing the cases of endometriosis

\section{KEYWORDS : Infertility, Laparoscopy, Endometriosis, Pelvic pain, ultrasound}

\section{INTRODUCTION}

Endometriosis is defined as a chronic and recurrent disease characterised by the presence and proliferation of endometrial glands and stroma outside the uterine cavity. It is responsible for varied and disabling symptoms and it also has an adverse impact on fertility. The prevalence of endometriosis in reproductive women is around $10 \%$ to $20 \%$ and endometriosis is the cause of infertility in $30 \%$ to $70 \%$ of patients coming for infertility investigation. 1,2

Adnexal mass is common among women of all age groups but commoner among women of reproductive age.endometriosis is a common cause of dysmenorrhoea and infertility .

The aetiology of endometriosis is complex and multifactorial. 3 Even though it is benign in nature; the majority of women do not improve if left untreated. The most common symptoms of endometriosis are dysmenorrhoea, dyspareunia, pelvic pain and infertility. 4

The surgical modalities include laparoscopy and laparotomy which re diagnostic as well as therapeutic.

The major challenge in endometriosis is its diagnosis and hence its treatement which can be medical or surgical.

\section{METHODS}

This study was performed at Grant Govt Medical College, Mumbai, Maharashtra, between June 2017 to dec 2020. The patients included in the study were in the age group 18 to 45 years with symptoms of dysmenorrhoea, dyspareunia, pelvic pain and infertility. Pelvic examination was done and clinical findings of POD tenderness with nodular surface, restricted mobility of uterus or fixed retroverted uterus with adnexal mass either unilateral or bilateral were noted. All the patients underwent ultrasound and the accuracy of ultrasound in diagnosing the adnexal mass as endometriosis was noted. Diagnostic standard three-trocar laparoscopy was done under general anaesthesia, with a $10 \mathrm{~mm}$ operating laparoscope inserted through an umbilical port and two $5 \mathrm{~mm}$ sheaths inserted in the lower abdominal quadrants lateral to the inferior epigastric artery. All the specimens retrieved were sent for histopathological (HPE) confirmation.

\section{RESULTS}

The prevalence of endometriosis in women, who were included in the study, was found to be $6.31 \%(n=12)$.

\begin{tabular}{|l|l|l|}
\hline Age distribution & $\begin{array}{l}\text { Histopathological proven } \\
\text { cases }\end{array}$ & percentage \\
\hline $20-30$ & 5 & $41.66 \%$ \\
\hline $30-40$ & 4 & $33.33 \%$ \\
\hline $40-45$ & 2 & $16.66 \%$ \\
\hline
\end{tabular}

The commonest presenting age was 20 - 30 years of age group.

The ultrasound diagnosis of endometriosis was found in $5.94 \%(n=12)$ with 2 cases showing bilaterality and 7 showing septations.

The commonest symptomatology was pain in abdomen ( $59.4 \%$ ) followed by menstrual complaints (14.35) amongst the 200 cases of adnexal masses that were studied.out of which $7.44 \%$ has dyspareunia and infertility

\begin{tabular}{|l|l|l|l|l|}
\hline Clinical findings & usg & $\begin{array}{l}\text { No of } \\
\text { cases }\end{array}$ & Hpr & $\begin{array}{l}\text { No of } \\
\text { cases }\end{array}$ \\
\hline $\begin{array}{l}\text { P/A - soft, no } \\
\text { guarding, rigidity } \\
\text { and tenderness }\end{array}$ & endometriosis & 11 & endometriosis & 11 \\
\hline $\begin{array}{l}\text { Palpable mass } \\
\text { per abdomen }\end{array}$ & Endometriosis & 1 & & \\
\hline $\begin{array}{l}\text { Per vagina } \\
\text { fornical mass or } \\
\text { tenderness }\end{array}$ & & endometriosis & 5 \\
\hline
\end{tabular}

When clinical examination findings were correlated with ultrasonography and histopathological findings , it was found that most of the cases of endometriosis had no significant clinical findings on per abdomen examination.

Association of USG \& HPR in cases of ENDOMETRIOSIS

Data: Number(\%)

\begin{tabular}{|l|l|l|l|}
\hline & HPR + & HPR - & Total \\
\hline USG + & $5(6.7 \%)$ & $1(100.0 \%)$ & 6 \\
\hline USG - & $1(93.3 \%)$ & $6(0.0 \%)$ & 7 \\
\hline Total & $6(100.0 \%)$ & $7(100.0 \%)$ & 13 \\
\hline
\end{tabular}


Statistical Test

Fisher Exact Probability Test

Fisher Exact Probability Test value $=0.03, \mathrm{DF}=1, \mathrm{~S}, \mathrm{P}=0.03$

Conclusion: Significant association between USG \& HPR in cases of endometriosis.

\begin{tabular}{|l|l|l|}
\hline Parameter & Value & $\begin{array}{l}\text { 95\% Confidence } \\
\text { Intervals }\end{array}$ \\
\hline Sensitivity & 83.33 & $35.88,99.58$ \\
\hline Specificity & 85.71 & $42.13,99.64$ \\
\hline Positive Likelihood Ratio & 5.83 & $0.92,37.08$ \\
\hline Negative Likelihood Ratio & 0.19 & $0.03,1.19$ \\
\hline Positive Predictive Value (PPV) & 83.33 & $44.03,96.95$ \\
\hline Negative Predictive Value (NPV) & 85.71 & $49.43,97.36$ \\
\hline Accuracy & 84.62 & $54.55,98.08$ \\
\hline
\end{tabular}

Graph

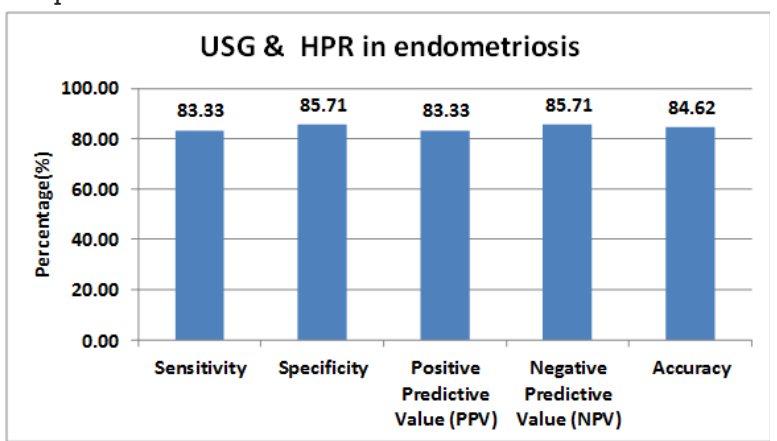

\section{DISCUSSION-}

TOMOGRAPHYEndometriosis is the presence of endometrial tissue outside of the endometrium and myometrium. It can involve a wide range of locations; including the ovaries, uterine ligaments, rectovaginal septum, cul-de-sac and pelvic peritoneum being the most common sites. The prevalence of the disease in women of reproductive age range up to $15 \% 5$

It is a benign, inflammatory, estrogen dependent condition in which tissue corresponding in every respect to the endometrium (glands and stroma) occurs outside the uterus in various parts of the body.

This ectopic endometrial tissue responds to cyclic ovarian hormones and bleeds as if it were located within the uterus. Endometrial cysts (endometrioma) may develop in these areas of bleeding. Small cysts are termed 'blebs' whereas larger ones, because of their contents (blood) and colour, are called chocolate 'cysts'. This type of cyst may occur singly, but more than one are generally seen. Because these cysts contain blood, they may contain internal echoes in the form of either many low-level echogenic structures or a dense echogenic "blob"

The term endometriosis was coined in 1927 by Dr John Sampson.

The "classical terrible triad " of symptoms of endometriosis include dysmenorrhea, dyspareunia and infertility.The cause of dyspareunia is mostly as a result of extension of endometriosis to uterosacral ligaments.

Non cyclic abdominal and pelvic pain of atleast 6 months duration is a characteristic feature.

Alpha sequence

A systematic laparoscopic inspection method called alpha sequence using a $300,10-\mathrm{mm}$ endoscope is important in complete pelvic assessment.

It begins from anterior abdominal wall, progresses to bladder , round ligaments, anterior and posterior surface of uterus , the uterosacral ligaments, posterior cul - de - sac , rectum sigmoid colon, then to left broad ligament, left oviduct ,left ovary and left ureters and then the right adnexa. Lastly it is proceded towards the appendix , distal ileum , cecum , gall bladder, liver, undersurface of diaphragm, stomach and omentum.

Cal25 levels may be moderately raised in endomteriosis , however it has more of prognostic than diagnostic value.

Endometriosis remains a diagnostic as well as a therapeutic impasse. It is considered as an enigma; hence it still intrigues researchers to addressing the cause and the management of the disease in every possible way. Endometriosis has a deep impact on quality of life, and developing a therapy that also improves fertility remains a challenge for gynaecologists. The exact mechanism by which it causes infertility is still unclear. The probable sequences of the causes of infertility in cases of endometriosis are by an altered peritoneal fluid composition as a result of hormonal, genetic and environmental factors. 6,7 One of the accepted theories is increased levels of prostaglandins, proteases, and cytokines and vascular endothelial growth factor (VEGF) in the peritoneal fluid. 8

The diagnostic utility of USG in endometriosis is limited, but it can give corroborative evidences when there are ovarian endometriomas. 9 however I this study a significant correlation was obtained between the ultrasound diagnosed cases and those confirmed on histopathology.

Transvaginal sonography does not make out endometriotic implants 10,1l. Endometriomas have a variety of appearances, ranging from an anechoic cyst to a cyst including diffuse low level echoes with or without solid components to solid appearing masses.

Although the ultrasonographic findings are sometimes imprecise, differentiation from functional hemorrhagic cysts or other echogenic cysts is in general possible by demonstrating multiple thick walls, homogeneity of the echogenic content and 12,multiplicity of the lesions 13. Also, the presence of punctate or linear bright echogenic foci in the wall of the cyst contributes to the diagnosis of an endometrioma.

Medical management is based on the basic principle of reducing inflammation, suppressing ovarian cycles and inhibiting the effect of oestrogen but its role in treatment of infertility is limited. Medical management can be used prior to surgery to decrease the size of endometriotic lesions and the extent of the operation.

The recent advances in operative laparoscopy have changed the view in the management of endometriosis with infertility. The laparoscopic treatment involves the identifying and removal of lesions by cauterization, fulguration or laser evaporation for minimal to mild disease, adhesiolysis, excision of deep lesions, cystectomy, drainage and coagulation for endometriomas of ovary (moderate to severe disease).

It is generally believed the prevalence of endometriosis is less common in India, and in our study in the Mumbai it was $6.31 \%$ of all the reproductive women studied. The most common site remain that in the pelvis with obliteration of cul-de-sac and ovarian endometriomas. The diagnosis can be well established in all cases by laparoscopy and HPE confirmation.

\section{CONCLUSIONS}

Patients with symptoms of dysmenorrhoea, dyspareunia, pelvic pain, infertility and clinical signs of cul-de-sac 
tenderness with nodular surface, restricted mobility of uterus or fixed retroverted uterus with adnexal mass along with USG findings should raise the suspicion of endometriosis in infertility patients. with the recent advances in ultrasound, it turns out to be an important method for diagnosing the endomeriotic cases. However results of this study are not statistically conclusive because of small sample size and further studies are required.

\section{REFERENCES-}

1. Eskenazi B, Warner ML. Epidemiology of endometriosis. Obstet Gynecol Clin North Am. 1997;24:235-58

2. Donnez J, Wyns C, Nisolle M. Does ovarian surgery for endometriomas impair the ovarian response to gonadotropin? Fertil Steril. 2001;76:662-5.

3. Burney RO, Giudice L. Pathogenesis and pathophysiology of endometriosis. Fertil Steril. 2012;98:511-9.

4. Carneiro MM, Filogônio I DDS, Costa LMP, De Ávila I, Ferreira MC. Accuracy of clinical signs and symptoms in the diagnosis of endometriosis. J Endometr. 2010;2:63-70.

5. Strathy JH, Molgoard CA, Coulam CB, Melton LJ. Endometriosis and infertility: a laparoscopic study of endometriosis among fertile and infertile women. Fertil Steril 1992;38:667-72.

6. Gilabert-Estelles J, Ramon LA, España F, Gilabert J, Castello R, Estelles A Expression of fibrinolytic components in endometriosis. Pathophysiol Haemost Thrombs. 2006:35:136-40.

7. Szczepa ska M, Ko lik J, Skrzypczak J, Mikołajczyk M. Oxidative stress may be a piece in the endometriosis puzzle. Fertil Steril. 2003;79:1288-93

8. Bedaiwy MA, Falcone T, Sharma RK, Goldberg JM, Attaran M, Nelson DR et al. Prediction of endometriosis with serum and peritoneal fluid markers: $\alpha$ prospective controlled trial. Hum Reprod. 2002;17:426-31.

9. Patel MD, Feldstein VA, Chen DC, Lipson SD, Filly RA. Endometriomas; diagnostic performance of US. Radiol. 1999;210(3):739-45.

10. Friedman H, Vogelzang RC, Mendelson EB, Nieman HL, Cohen M. Endometriosis detection by US with laparoscopic correlation. Radiology 1985: 157:217-20.

11. Kupfer MC, Schwimer SR, Lebovic J. Transvaginal sonographic appearance of endometriomata: spectrum of findings. J Ultrasound Med 1992; 11:129-33.

12. Osmors R. Sonographic evaluation of ovarian masses and its therapeutic implications. Ultrasound Obstet Gynecol 1996; 8:217-22.

13. Pascual MA, Treurra F, Lopez-Marlia L, Obeda A, Grases PJ, Dexeus S. Role of color Doppler ultrasonography in the diagnosis of endometriotic Cyst. J Ultrasound Med 2000; 19:695-9. 